\title{
Study on the influence line of plane statically truss by MAPLE
}

\author{
Ming Bo Zhao ${ }^{1, a}$,Xiao Hui Luo ${ }^{1, b}$ \\ ${ }^{1}$ (School of Civil Engineering and Architecture, Southwest University of Science and \\ Technology,MianYang 621010,China) \\ azmwave@126.com, ${ }^{b}$ lang.lxh@163.com
}

Keywords: MAPLE, plane statically determinate truss, influence line, Accurately calculate Abstract. This paper elaborate the application of MAPLE in the calculation of the influence line of the plane statically determinate truss, accurate calculation of the axial force of a plane statically determinate truss by symbolic computation in MAPLE, that we can get the influence value by axial force to plot influence line from axial force. It can be serviced for teaching, scientific research, engineering practice and question bank.

\section{Introduction}

The influence line of plane statically determinate truss is calculated by VBA programming in EXCEL from reference ${ }^{[1]}$. It requires professional programming knowledge, which can only be calculated by numerical calculation, and the result of calculation is expressed in decimal. The universal program on MAPLE for axial force of plane statically determinate truss is given from reference $^{[2]}$, using symbolic computation and accurate calculation of the software MAPE, we can program calculate the influence coefficients of plane statically determinate truss, and plot the influence line of the plane statically determinate truss.

\section{Data structure of plane statically determinate truss}

Each programming language has its own character and data structure, In order to describe the basic information of plane statically determinate truss, Only four sets of data is needed, that is:

(1)、Coordinate data XY: two dimensions matrix, column $1 \& 2$ is $\mathrm{x} \& \mathrm{y}$ coordinates of the nodes, the row number of matrix is equal to the number of nodes.

(2)、Element data NE: two dimensions matrix, column $1 \& 2$ express starting and ending points of a element, The row number of matrix is equal to element number.

(3)、Constraint data NC: two dimensions matrix, column $1 \& 2$ of the matrix is the node number of constraint and direction number, 1 as $\mathrm{x}$ direction and 2 as y direction.

(4)、Moving load data NP: using row vector description, The element is the node number of the moving load moving path.

\section{How to handle the unit moving load}

There are many ways to handle the unit moving load, in order to quote original code from reference ${ }^{[2]}$,along the moving path of unit moving load, replacing the unit moving load with a symbol $F_{k}$ (concentrate force), the load can be given to truss nodes. That is to say, if y direction of the node has unit moving load, In the y direction of the node to apply a down concentrate force $F_{k}$.

\section{Determinate Influence coefficient}

Using original code from reference ${ }^{[2]}$, the axial force $N_{i, j}$ of the truss is the linear combination about $F_{k}$ can be calculated. The axial force $N_{i, j}$ can be expressed as:

$$
N_{i, j}=\sum a_{k} F_{k}
$$


It means, when the pole $i-j$ of truss has down concentrate force $F_{k}$ on node k of y direction,the axial force of pole $i-j$ is $a_{k} F_{k}$,when $F_{k}=1, a_{k}$ is the influence coefficients. the influence line of pole $i-j$ can be obtained by using a broken line from each points $a_{k}$.

\section{Drawing diagram}

Not only does MAPLE have the powerful symbolic computation and numerical calculation function,but aols the drawing function is powerful,you can write a program to draw the plane truss diagram and influence line diagram with a little program code limited by space, no details.

\section{The influence line of plane statically determinate truss}

Using original code from reference ${ }^{[2]}$, when the unit moving load move along node1,2,3,4,5, the axial force of each pole of the truss in Fig. 1 is shown in table 1. we can get the axial force of pole 3-6 is $N_{3,6}=\frac{\sqrt{13}}{6} F_{2}-\frac{\sqrt{13}}{4} F_{3}-\frac{\sqrt{13}}{6} F_{4}$ from table 1,that is, the relationship between the axial force $N_{3,6}$ and each unit moving load $F_{k}$ is $N_{3,6}=0 \times F_{1}+\frac{\sqrt{13}}{6} F_{2}-\frac{\sqrt{13}}{4} F_{3}-\frac{\sqrt{13}}{6} F_{4}+0 \times F_{5}$, so the influence coefficient of pole 3-6 is $\left(0, \frac{\sqrt{13}}{6},-\frac{\sqrt{13}}{4},-\frac{\sqrt{13}}{6}, 0\right)$, the influence lines of pole 3-6 is shown in Fig. 2. and the influence lines of pole 3-7 is shown in Fig.3. we can get the influence line of the rest of the pole and supported force from table 1 .using original code from reference ${ }^{[2]}$, and only one symbolic computation,we can get the axial force and supported force of the truss, which we can use to get the influence coefficients and plot the diagram of influence line.

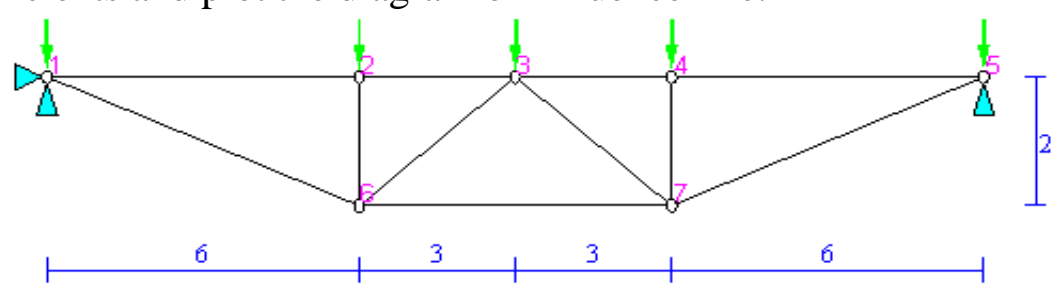

Fig.1 Plane statically determinate truss

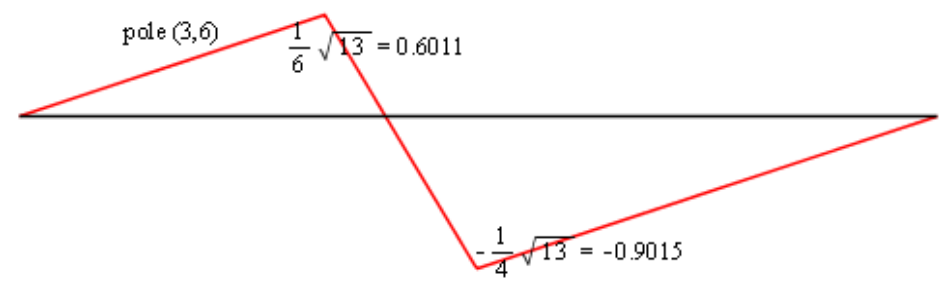

Fig.2 Influence line of pole 3-6

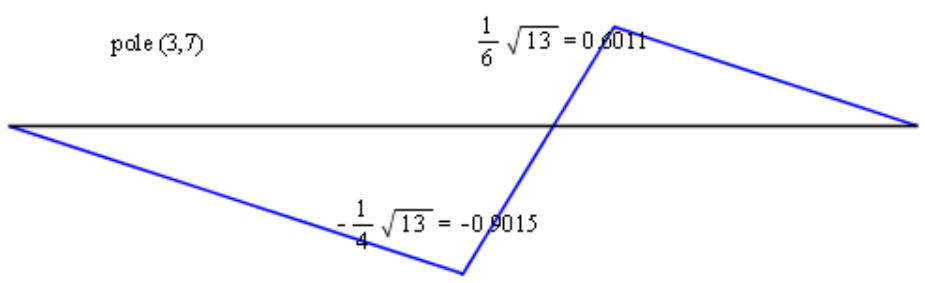

Fig.3 Influence line of pole 3-7 


\section{Conclusions}

MAPLE programming which has its special advantages is used to calculate the axial force of the plane statically determinate truss by symbolic computation, it can be used to calculate the influence value of plane statically determinate truss, and drawing influence lines, service for teaching, scientific research, engineering practice and question bank construction.

Table 1. The axial force and the supported force of the truss as shown in Fig.1

\begin{tabular}{|l|}
\hline$N_{1,2}=-2 F_{2}-\frac{3}{2} F_{3}-F_{4}$ \\
\hline$N_{1,6}=\frac{2}{3} \sqrt{10} F_{2}+\frac{1}{2} \sqrt{10} F_{3}+\frac{1}{3} \sqrt{10} F_{4}$ \\
\hline$N_{2,3}=-2 F_{2}-\frac{3}{2} F_{3}-F_{4}$ \\
\hline$N_{2,6}=-F_{2}$ \\
\hline$N_{3,4}=-F_{2}-\frac{3}{2} F_{3}-2 F_{4}$ \\
\hline$N_{3,6}=\frac{1}{6} \sqrt{13} F_{2}-\frac{1}{4} \sqrt{13} F_{3}-\frac{1}{6} \sqrt{13} F_{4}$ \\
\hline$N_{3,7}=-\frac{1}{6} \sqrt{13} F_{2}-\frac{1}{4} \sqrt{13} F_{3}+\frac{1}{6} \sqrt{13} F_{4}$ \\
\hline$N_{4,5}=-F_{2}-\frac{3}{2} F_{3}-2 F_{4}$ \\
\hline$N_{4,7}=-F_{4}$ \\
\hline$N_{5,7}=\frac{1}{3} \sqrt{10} F_{2}+\frac{1}{2} \sqrt{10} F_{3}+\frac{2}{3} \sqrt{10} F_{4}$ \\
\hline$N_{6,7}=\frac{3}{2} F_{2}+\frac{9}{4} F_{3}+\frac{3}{2} F_{4}$ \\
\hline$X_{1}=0$ \\
\hline$Y_{1}=F_{1}+\frac{2}{3} F_{2}+\frac{1}{2} F_{3}+\frac{1}{3} F_{4}$ \\
\hline$Y_{5}=\frac{1}{3} F_{2}+\frac{1}{2} F_{3}+\frac{2}{3} F_{4}+F_{5}$ \\
\hline
\end{tabular}

\section{References}

[1].M.B.Zhao, Influence Line Analysis of the Plane Trusses Based on EXCEL, Progress in Structures[J], 166-169 (2012) 927-930.

[2] Ming Bo Zhao, Algebraic solution of plane statically determinate truss by MAPLE [J], Shanxi architecture, 6(2006)353-354 (in chiness). 